\title{
Research of "Digital Signal Processing” experiment Based on LabVIEW
}

\author{
Yang Zhonglin \\ College of Electrical Engineering \\ Navy University of Engineering \\ Wuhan, China \\ blueduny@sina.com
}

\author{
Hou Xinguo \\ College of Electrical Engineering \\ Navy University of Engineering \\ Wuhan, China \\ Hxinguo2008@126.com
}

\begin{abstract}
In this paper, the characteristics of the Digital Signal Processing course and the LabVIEW software were introduced, and the advantages of application the LabVIEW software in Digital Signal Processing course experiments were pointed out also. Three examples of application the LabVIEW software in Digital Signal Processing course experiment were given. Practice has proved that the application the LabVIEW software in the Digital Signal Processing course experiments can improve students' interest in learning and achieve very good teaching effect.
\end{abstract}

Keywords-LabVIEW; Digital Signal Processing; Course experiments; Sampling theory

\section{INTRODUCTION}

Digital signal processing has been widely used in communication engineering, electronic information engineering, radar engineering, biological engineering and many other fields, which was extremely important in today's high-tech field ${ }^{[1-4]}$.

Therefore, digital signal processing has become one of the main specialized courses in many universities now. However, the digital signal processing was a strong theoretical discipline, the core of which is the algorithm. The related theories were very abstract, and it deals with a lot of mathematic formulas which were difficult to understand. The students have not the visual results in the learning process, which makes the students' understanding of the theories very superficial. So the students' enthusiasm about study was greatly reduced, which affects the teaching effect in the great extent ${ }^{[5-7]}$. How to effectively combine theories with practice and increase the learning interest of students is an urgent problem in the teaching course of digital signal processing. In order to help the students to learn the course, showing the related theories in the intuitive way was very necessary. So, teaching experiment was an indispensable link.

However, most of the existing experimental methods were implemented by using the high-level language programming simulation software, such as MATLAB. The simulation experiments require students to learn programming software. As students were not interesting in the computer simulation experiments which were finished by edited the text code, so the teaching effect is not well. And in the case of insufficient budget, teachers could not buy a lot of experimental instrument. So, the LabVIEW software was introduced into the experimental teaching of digital signal processing and become a direction of experiment reform of digital signal processing ${ }^{[8-10]}$.

\section{THE BRIEF INTRODUCTION OF THE LABVIEW SOFTWARE}

The LabVIEW software was an graphical integrated development environment based on the graphical programming language --Graphics Language which was developed by the National Instruments. In the LabVIEW software, the control panels of the conventional instruments have been supplied and do not need complex programming code. They can be used to complete the requirements of users by using the Graphics Language. The programs of the LabVIEW mainly include two parts of front panel and program panel. The front panel is used to place a variety of controls, including the input control, display control, regulation control, which can be arranged in the user interface by dragging the corresponding icon simply. The program panel is used for placing various functions, including signal generation, signal analysis, data acquisition, and so on. The more complex functions can be realized by combining the function module. The complex programming works on traditional development environment do not need. We can easily and quickly develop a corresponding application interface by using the graphical controls ${ }^{[11,12]}$.

\section{THE ADVANTAGES OF EXPERIMENT TEACHING BASED ON THE LABVIEW SOFTWARE}

The Graphics Language of can make students focus on the theoretical knowledge, rather than the programming details, so that the students can develop complex applications in a very short period of time, and put the theoretical knowledge into the graphic, which can deepen the students' understanding for the theoretical knowledge, and improve the quality of teaching. Specifically, the application of LabVIEW in the experimental teaching of digital signal processing course has the following advantages:

(1) The LabVIEW software use the graphical programming language and basically do not need write code, which was replaced by the program diagram. And the LabVIEW software has a variety of powerful functions, which are convenient and flexible to use. This graphical development environment can reduce the difficulty of programming and improve the work efficient.

(2) The signal processing suite of LabVIEW can be used for joint time-frequency analysis, digital filter design, high accuracy of spectrum analysis and wavelet design and filter group design. So the LabVIEW software was very suitable to be used in the field of the digital signal processing. 
(3) The virtual instruments based on the LabVIEW software not only can replace the traditional measure instruments, but also supply some functions which the traditional measure instruments can not supply. It has brought the huge change for experiment teaching of digital signal processing.

\section{THE EXAMPLES OF THE EXPERIMENT BASED ON LABVIEW}

\section{A. The sampling theory experiment based on LabVIEW}

The sampling theorem is a very important content of digital signal processing course. It is a bridge connecting the discrete time signal and the continuous time signal, for providing a theoretical basis of their mutual transformation. The main contents of the sampling theorem is ${ }^{[13]}$. Let $x(t)$ be a band-limited signal with $X(j \omega)=0$ for $|\omega|>\omega_{\mathrm{M}}$. Then $x(t)$ is uniquely determined by its samples $x(n T)$, if $\omega_{\mathrm{s}}>2 \omega_{\mathrm{M}}$, where $\omega_{\mathrm{s}}=2 \pi / T$.

The sampling theorem is abstract and difficult to understand. In order to help students better understand the sampling theorem, using the LabVIEW graphical programming language to design a experimental program of continuous signal sampling not only can deepen students' understanding of sampling theorem, but also can improve their interest in the digital signal processing course.

The figure 1 is the program panel of the experimental program. The continuous signal includes two different frequency sine signals, and each sinusoidal signal can be generated by the simulation signal VI. The spectrum analysis of the signal is completed by the FFT spectrum of VI. The sampling was finished by the multiplication by of the continuous signals and rectangular pulse sequence. The sinusoidal signal frequency, amplitude and the sampling frequency can be set by users themselves.

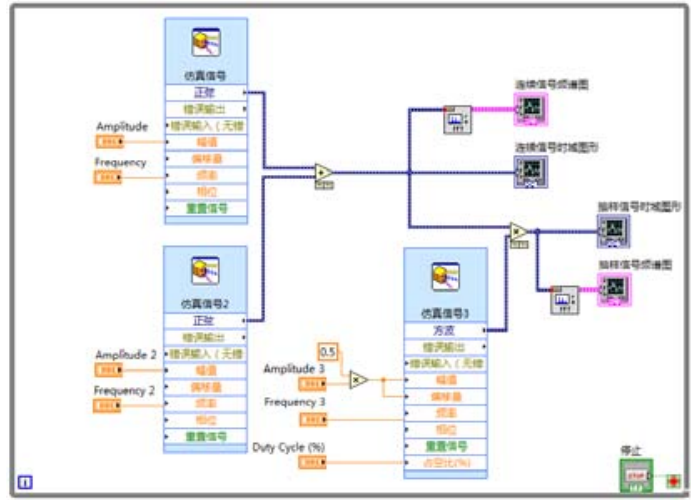

Figure 1. The program panel of the sampling theorem experiment

The figure 2 is the front panel without spectrum aliasing, and the figure 3 is The front panel with spectrum aliasing. In the figure 2, the continuous signal contains $50 \mathrm{~Hz}$ and $200 \mathrm{~Hz}$ of two frequency components. The sampling frequency is $500 \mathrm{~Hz}$. So the sampling frequency is more than two times the highest frequency of continuous signal. After sampling, the signal spectrum has not the spectrum aliasing. The original continuous signal can be recovered through a low pass filter. In the figure 3, the Frequency components of original continuous signal have not been changed, but the sampling frequency was changed to $200 \mathrm{~Hz}$. Two times the sampling frequency is less than the maximum frequency of original continuous signal. After sampling, the signal spectrum has aliasing distortion, so the original signal can not be restored. Using the above program, the sampling process can be observed by the images which can help the students to deepen the understanding of sampling theorem.

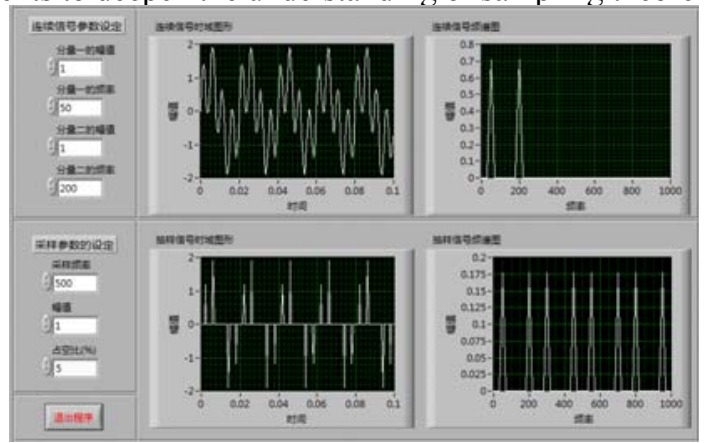

Figure 2. The front panel without spectrum aliasing

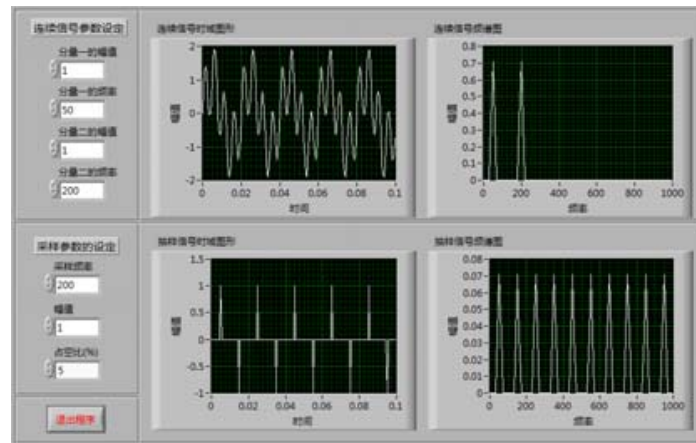

Figure 3. The front panel with spectrum aliasing

\section{B. The digital filter experiment based on LabVIEW}

In the experiment of digital signal, the filter is often used. In the traditional experiment box, the filter often are analog filter, the performance of which is not stable, and the parameter range of which is restricted by hardware and can not be regulated arbitrarily. The digital filter which be designed by using the LabVIEW software can be adjusted without increasing the cost, and various parameters can also be modified according to the user's demand arbitrarily.

In this experiment, It was supposed that the signal was mixed with high-frequency noise in the transmission process, so we hoped that the high-frequency noise signal can be filtered through the low-pass filter. The front panel is shown in figure 4 and the program panel is shown in figure 5.

In the Figure 4, it can be seen that when the noise signal frequency is higher than $100 \mathrm{~Hz}$ and the cutoff frequency of the low-pass filter is $30 \mathrm{~Hz}$, the high-frequency noise signal has been filtered and the sine signal has been restored. 


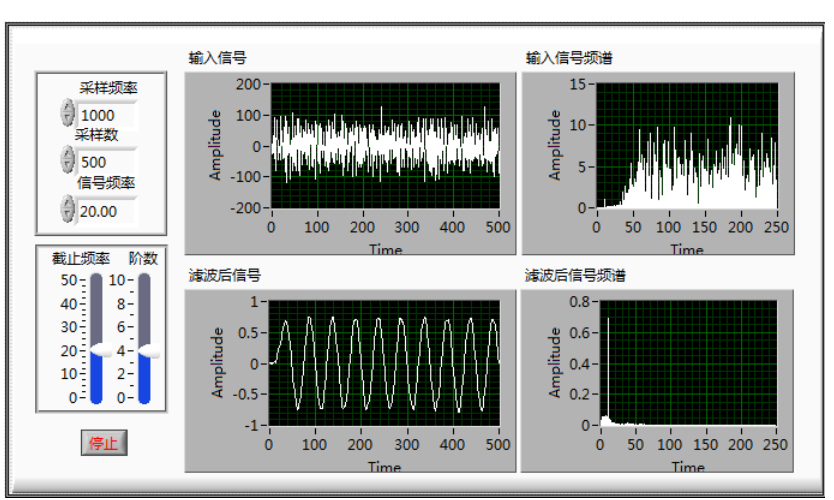

Figure 4. The front panel of the filter experiment

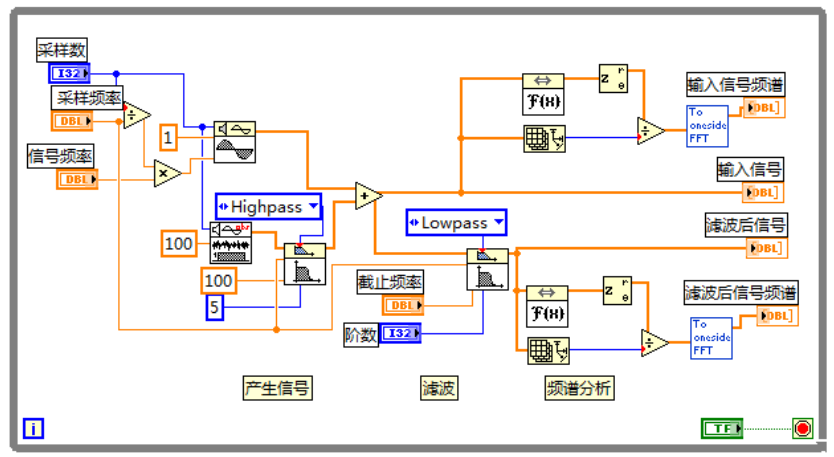

Figure 5. The program panel of the filter experiment

\section{The window function experiment based on LabVIEW}

In the actual measurement, the number of the value for the sampling signal is limited. When analyzing signal spectrum with FFT, we assume that the original continuous signal is a periodic signal, the first period is sampled signal, and the original signal is periodic sampling signal replication. Because the signals of contiguous period are not continuous, it will cause the "spectral leakage" phenomenon, namely as a frequency energy leak to other frequency.

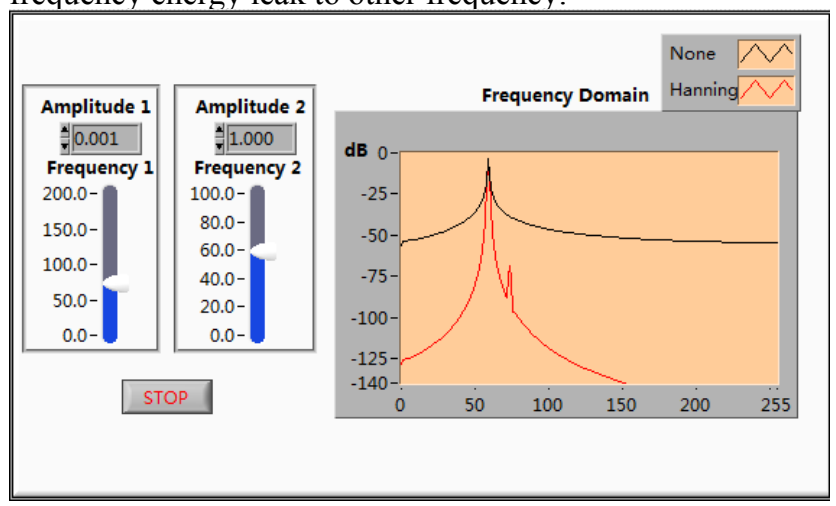

Figure 6. The front panel of the window function experiment

When the amplitude of a frequency component of signal is relatively small, due to spectrum leakage, it is difficult to distinguish the smaller amplitude signals when the FFT analysis was done directly. But if we add a proper window function to the signal and then do FFT, it is easy to distinguish the smaller amplitude signals. The front panel is shown in figure 6 and the program panel is shown in figure 7.

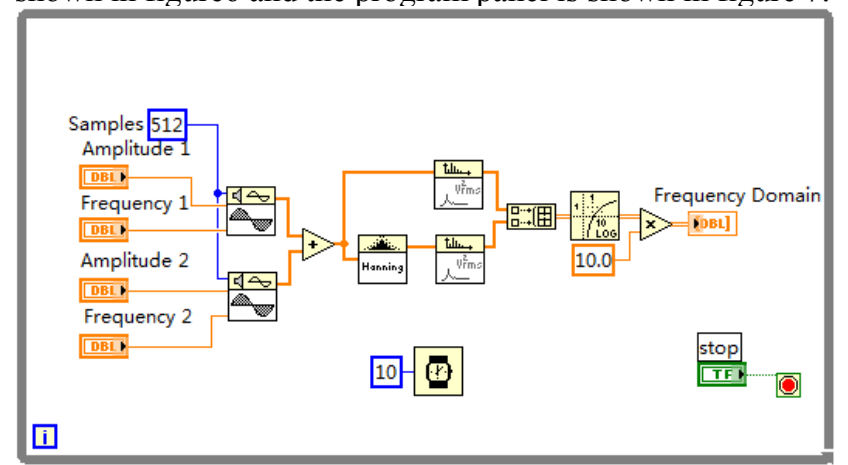

Figure 7. The program panel of the window function experiment

In the Figure 6, it can be seen that when the ratio of two sinusoidal signal' amplitudes is above 1000 , it was very hard to see the smaller amplitude signal from spectrum of the mixed signal without the hanning window, while the smaller amplitude signal is very obvious in the spectrum of the mixed signal after Hanning window.

\section{CONClusion}

Through the above examples, we can see that the LabVIEW's graphical language can be used to develop the digital signal processing programs which are complicated in a short period of time. At the same time, in the course of the experiment the students can also modify the program parameters and analysis the results, which enhance the student's understanding about the theoretical knowledge and improve their learning interest. It has been proved that the good teaching effect can be achieved through the using of LabVIEW software in the digital signal processing experiment teaching.

\section{REFERENCES}

[1] Zheng Nanling, and Chen Hong, Diginal Signal Processing, Beijing: Qinghua university Press, 2007.

[2] Chen Peiqing, Diginal Signal Processing, 3th ed., Beijing: Qinghua university Press, 2007.

[3] Yin Weimin, Ouyang Hua, and Qian Mei, Diginal Signal Processing, Beijing: Machinery Industry Press, 2011.

[4] Yao Tianren, and Jiang Taihui, Diginal Signal Processing, 2nd ed. Wuhan: Huazhong university of science and technology

[5] Wu Ling-xi. and Liu Wang-dong, "Application of window function in the design of digital filter," Journal of Xiangtan Normal University, vol. 24,Sep. 2002, pp. 57-60

[6] Li Lu, Shi zhenwei, and Zhou Fu-gen, "Comprehensive Experimental Design of Amplitude Modulation and Demodulation Based on Matlab/Simulink," Research and Explloration in Laboratory, vol. 30, Jan. 2011, PP. 96-99.

[7] Zhang Deng-qi, Zhou Ting, and Li Bin, "Realization and Simulation of Digital Filter Frameworks Based on Matlab," Journal of Hunan Institute of Science and Technology, , vol. 21, Sep. 2008, PP. 19-22.

[8] Chen Xing-wen, Li Min, and Liu Yan, "LabVIEW application of practice teaching in digital signalproessing," Journal of Science of Teachings' College and University, vol. 27, Nov. 2007, PP. 90-92. 
[9] Chen Ling-ling, Liu Yang, and Qin Li-min, "Design of DSP experimental platform based LabVIEW," Jounal of Jilin Institute of Chemical Technology, vol. 28, Nov. 2011, PP. 66-69

[10] Quan Xiaoli, Zhou Nanquan, and Li Shuang, "Building Virtual experiment of digital signal processing based on LabVIEW," Experimental Technology and Management, vol. 28, Oct. 2011, PP. 82-44.
[11] Yue Hongwei, "Implementation of DSP virtual experiment system based on LabVIEW," Experimental Technology and Management, vol. 28, Agu. 2011, PP. 70-72.

[12] Zhang Meng, "Construction and Implementation of Virtual Teaching Experiment Platform Based on LabVIEW," Journal of ChangChun University, vol. 21, Agu. 2011, PP. 19-22.

[13] Alan V. Oppenheim, Discrete-Time Signal Processing, 2nd ed., Prentice Hall, 1999. 